\title{
MRI Brain Tumour Segmentation Using Hybrid Clustering and Classification by Back Propagation Algorithm
}

\author{
Malathi M*, Sinthia P
}

\begin{abstract}
Generally the segmentation refers, the partitioning of an image into smaller regions to identify or locate the region of abnormality. Even though image segmentation is the challenging task in medical applications, due to contrary image, local observations of an image, noise image, non uniform texture of the images and so on. Many techniques are available for image segmentation, but still it requires to introduce an efficient, fast medical image segmentation methods. This research article introduces an efficient image segmentation method based on $\mathrm{K}$ means clustering integrated with a spatial Fuzzy $\mathrm{C}$ means clustering algorithms. The suggested technique combines the advantages of the two methods. $\mathrm{K}$ means segmentation requires minimum computation time, but spatial Fuzzy $\mathrm{C}$ means provides high accuracy for image segmentation. The performance of the proposed method is evaluated in terms of accuracy, PSNR and processing time. It also provides good implementation results for MRI brain image segmentation with high accuracy and minimal execution time. After completing the segmentation the of abnormal part of the input MRI brain image, it is compulsory to classify the image is normal or abnormal. There are many classifiers like a self organizing map, Back propagation algorithm, support vector machine etc., The algorithm helps to classify the abnormalities like benign or malignant brain tumour in case of MRI brain image. The abnormality is detected based on the extracted features from an input image. Discrete wavelet transform helps to find the hidden information from the MRI brain image. The extracted features are trained by Back Propagation Algorithm to classify the abnormalities of MRI brain image.
\end{abstract}

Keywords: K means clustering- Fuzzy C means clustering- spatial fuzzy C means- discrete wavelet transform

Asian Pac J Cancer Prev, 19 (11), 3257-3263

\section{Introduction}

The process of separating of an image into many small regions is called image segmentation. The main objective of the segmentation is to modify the representation of an image, which makes the image to be more meaningful and helps to analyse easily. Hence the image segmentation plays a very important role in the medical field in order to identify the abnormality of the cells in our body. The proposed research work helps to identify the abnormality of a brain image. Image segmentation helps in the medical field to identify the region of interest and to examine the anatomical structure of body parts. The abnormality of the brain is identified by the researchers and physicians using various brain imaging techniques. Now a days invasive and safe imaging methods are used in medical field.

Ciulla et al., (2015) described many types of imaging, in which CT and MRI are the two important types of brain imaging methods. Hamamci et al., (2012) described the segmentation is the most important step in medical images for radiological or computer aided diagnosis. The segmentation refers, it is the process of splitting the image into small region based on some predefined similarity criterion. Hence the segmentation is one of the pixel classification methods based on similarity principle.

Image segmentation broadly classified into three categories like edge based method, region based method and pixel based method. The first method uses the edge information to define boundaries of the object. Further the boundaries are studied and changed, if necessary to produce closed regions of the objects in the image. The pixel based method uses the histogram based statistical features helps to form the closed regions belonging to the object in the image. The region based segmentation uses the some predefined similarity principle to classify the pixels of an image in order to form the closed region. After the identification of the closed regions the different types of statistical features are estimated like variance, entropy, mean and texture. Dimitri and Unser (2008) described the extracted features helps to characterize the closed regions which are extracted from various types of segmentation. Abdel-Maksouda et al., (2015) proposed method uses the hybrid clustering for MRI brain lesion segmentation, which helps to segment tumour from MRI brain image.

Zhang et al., (2015) developed new computerized 
imaging techniques for image reconstruction, processing and analysis methods for easy understanding and interpretation of medical images. This kind of mapping help the physicians to take medical decisions via physician and computer interaction. The sensitivity and specificity of radiological test is improved by new intelligent and quantitative based analysis. This leads to bring a various medical imaging moadalities like X-ray, CT, MRI, SPECT, PET and ultrasound with the help of the above mentioned methods. It is easy to generate the physiological structure of digital images. It also provides the characetersitics of hidden information by using computer, which is not possible to see with planar imaging methods. Clatz et al., (2015) discussed several techniques MRI is one of the good imaging technique to provide the tissue chracterisitics. Brain is the important organ of our human body, Because of its complex structure, the anatomy of the brain is scanned at MRI or CT, but the MRI is more suitable technique than the CT for diagnosis. Because it does not use the harmful radation.

\section{Brain tumour}

Brain tumour is one of the main reason for death. The early detection of tumour helps to increase the life time of the patient. Hence medical field needs to develop a new computer based segmentation methods with some preferred properties like minimum user interaction, fast computation and robust segmentation results. Ism et al., (2016) discussed there are several segmentation methods such as histogram based method, edge based method, region based method (Region splitting, growing and merging) and clustering method (Fuzzy C means clustering, K-means clustering, Mean shift and exceptation maximization). The selection of a suitable segmentation method for all types of image is a challenging task. But there is no universal accepted method for image segmentation.

Based on extensive literature review, clustering is one of the best image segmentation for MRI brain images. Clustering is performed in two ways on either partioning or by grouping pixels. Matthew et al., (1998) suggested the entire image is subdivided into small region based on some pre defined criteria, similarly in grouping the pixels are grouped together based on similarity property. There are many clustering methods are available for medical images such as $\mathrm{K}$ means clustering, Fuzzy $\mathrm{C}$ means clustering. The selection of suitable types of clustering method is a challenging task. Clustering is one of the preprocessing method for the classification of medical images.

\section{Related Work}

Medical image segmentation is one of the most important topic in medical field. Many researchers developed various segmentation method for image segmentation. Abdel-Maksouda et al., (2015) proposed a brain tumour segmentation method based on $\mathrm{K}$ means clustering and fuzzy $\mathrm{C}$ means. One of the simple and most suitable method for brain tumour segmentation is $\mathrm{K}$ means clustering method. K means clustering calculates the tumour region with minimal execution time. But in the case of malignant tumour this method provides improper segmentation result. Fuzzy $\mathrm{C}$ means is other kind of segmentation of bain tumour from MRI brain images. But this method is most suitable for noise free images. But it needs long iterative time for brain tumour segmentation. Hence the author combines K means and fuzzy $\mathrm{C}$ means clustering to provide new method called KIFCM (K means Integrated with Fuzzy C means). The implemented method uses the features of above two methods to overcome the long iteration time and improve the accuracy of segmentation.

The Juang and Wub (2010) proposed method uses colour converted segmentation algorithm with $\mathrm{K}$ means clustering in order to find the exact lesion size. The method converts the input gray image into colour space images. Hence the proposed method helps the pathologist to find exact lesion size.

The Islam et al., (2013) implemented brain tumour segmentation method based on feature extraction of brain MRI images. The complex appearance of the brain needs a new sophisticated model to formulate the features of MRI brain image. It uses mBm (multifunctioned Brownian motion method) and followed by novel algorithm to segment the spatially varying multifractal features. The implemented method also uses the adaboost algorithm to segment the texture features. The algorithm helps to assign the weights for classifier and to improve the classification efficiency.

Fuzzy C means clustering is one of the segmentation method used in earlier decades. Ramathilagama et al., (2011) implemented a method is used to preserve more information from the original image than the other segmentaion methods. Intensity inhomogeneity is one of the major computer based segmentation algorithms used to differentiate the borders between the tissues in medical images. But in conventional fuzzy $\mathrm{C}$ means algorithm utilize the spatial information for MRI brain tumour segmentation. Hence the author proposed a new method to obtain the spatial information for tissue based segmentation using modified Fuzzy C means.

Madhukumar and Santhiyakumari (2015) author implemented a method which performs the qualitative comparision of Fuzzy $\mathrm{C}$ means and $\mathrm{K}$ means segmentation using histogram guided initialization in order to differetinate the different tissue of tumour edema complex MR brain images. The method is implemented to segment graymatter, white matter, cerebro spinal fluid Necrotic focus of gliblastoma-multiforme (GBM) and the perifocal vasogenic edema from preprocessed T1 contrast axial plane MR images. From the implemented work it is identified that the $\mathrm{K}$ means identifies all six classes while Fuzzy c means identifies three classes. From the above literature review it is identified that most of the research work utilizes $\mathrm{K}$ means and fuzzy $\mathrm{C}$ means clustering method for segmentation of brain tumour MRI brain images. The author Ism et al., (2016) employed a deep learning method for automatic brain tumour segmentation. The article absolutely describes the various segmentation, like manual, semi automatic segmentation, full automatic segmentation and its challenges

The author Mohana et al., (2014) offers the complete 
review of denoising methods for the acquired MRI image may be affected by artifacts and noise. It can be removed using a variety of technologies like wavelet transforms, Curvelet transforms, filters. Generally the acquired image transformed into wavelet domain in order to obtain wavelet co-efficient thresholding is one of the operation helps to remove noise in the wavelet domain. Then IDWT is used to produce the denoised image. The performance of the various methods is analysed with the help of PSNR

Xing et al., (2016) implemented a method to find the brain tumour from the histopathologyspeciman for early detection. Because of its complex nature it is difficult to find the wound from the histopathology specimen. Hence Convolutionla neural network helps to segment the wound automatically by preserving the shape of image.

Yang et al., (2015) implemented a automatic brain tumour segmentation using magnetic resonanace spectroscopy via pattern recognition. The author combines Discrete wavelet Transform with unsupervised learning to remove wavelet features from the MRS signal. The combination of DWT based whole spectral analysis along with unsupervised learning improve clustering efficiency.

\section{Materials and Methods}

\section{Existing Method}

Finding the proper choice of the segmentation algorithm for the given applications is a difficult task in medical image segmentation. A novel method is introduced which combines any two segmentation methods to obtain the exact segment of lesion from MRI brain image. The process of combining two segmentation algorithms is called as hybrid segmentation. The main objective of combining the various algorithms is to remove the drawbacks of two different methods and to improve the accuracy of segmentation. The existing method combines $\mathrm{K}$ means and Fuzzy $\mathrm{C}$ means algorithms in order to segment the tumour from brain images.

\section{Proposed Method}

Many researchers proposed a new method of hybrid algorithm for segmentation. The proposed work implemented using MATLAB. It is the process of combining the two different kinds of algorithm to perform segmentation of medical images. This combination of two algorithms helps to obtain the advantages of the two methods. Till now the research work demonstrates the three different algorithm K-means, Adaptive K-means, Spatial Fuzzy c-means algorithm. The proposed method combines the $\mathrm{K}$ means algorithm along with a spatial fuzzy-c means algorithm in order to get the higher accuracy tumour detection and reduce the execution time required for segmentation process. Based on extensive literature review it is identified that clustering is one of thesuitable method to perform segmentation. Among several clustering method $\mathrm{K}$ means is chosen for the following reasons

- K means Practically work well even some assumptions are broken

- It is simple, easy to implement
- Easy to interpret the clustering results

- Fast and efficient in terms of computational cost

In $\mathrm{k}$ means algorithm the proper selection of $\mathrm{k}$ value is an important parameter in the tumour area calculation. But in the presence of noise and artifacts it produces poor segmentation result. The implementation of the algorithm for large data set is not possible and performs the incomplete segmentation for the type of malignant tumour. The other system is fuzzy C-means segmentation, it holds more useful information from the original image in order to detect the malignant tumour. But it take long time to complete the process. FCM does not use the spatial information during tumour detection and classification of textures. But in K-means the pixel which corresponds to any one of the clusters, unlike in SFCM the pixel which is capable to move to all clusters. SFCM relates all the spatial information into the membership function in order to perform clustering. Spatial function relates the summation of the value of the membership function in the neighbourhood of every pixel under consideration. SFCM is the most suitable method to process the overlapping gray scale intensities.

Xiang et al., (2002) proposed to overcome the disadvantage of the two methods can overcome by combining two different algorithms is called hybrid segmentation. The proposed system that utilizes the two algorithms combinable in order to obtain the unique features of the two methods. Logeswari and Karnan (2010) discussed many artificial neural network methods for classification of abnormalities for MRI brain image. Based on detailed review of many articles Sachdeva et al., (2016) proposed a support Vector machine and Artificial Neural Network for brain tumour classification of MRI brain image using active contour segmentation.

The classification of the subnormal region is classified based on applying various extraced features to train the SVM classifier. After performing the segmentation it is necessary to classify the tumour portion is benign or malignant. To perform these various kinds of classifiers are available like Support vector Machine, Back Propagation Algorithm and Self Organizing Map. Among all these classifier the proposed work utilizes Back Propagation Algorithm to classify the abnormal tissue. Because the BPA is one of the standard and easiest algorithm to implement. By initializing the weights properly the computation time required and consequently speed for the classification is improved.

\section{Results}

\section{Experimental Results} Image Preprocessing

After acquisition of images, the segmentation process begins with preprocessing. It is the compulsory step in segmentation in order to eliminate noise and artifacts from the acquired MRI image. The salt and pepper noise is added to the gray scale image by the amount of 0.02 . Salt and pepper noise has to be replaced with the help of the median filter. The preprocessing can be performed with various filters like median, adaptive, weighted filter. Among the various filters, median filter is used for brain 
Table 1. SFCM Output for 3 Sample Images

\begin{tabular}{|c|c|c|c|c|c|c|}
\hline S.No & Image & Result image & Area $\mathrm{mm}^{2}$ & Defected cells & Time Sec & Iterations \\
\hline 1 & & & 19.228 & 5695 & 8.7499 & 100 \\
\hline 2 & & & 18.747 & 5043 & 8.7479 & 100 \\
\hline 3 & & 3 & 10.85292 & 1690 & 6.5126 & 69 \\
\hline
\end{tabular}

Table 2. KISFCM Output for 3 Sample Images

\begin{tabular}{|c|c|c|c|c|c|c|}
\hline S.No & Image & Result image & Area $\mathrm{mm}^{2}$ & Defected cells & Time Sec & Iterations \\
\hline 1 & & 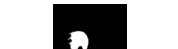 & 14.8616 & 3169 & 6.6995 & 76 \\
\hline 2 & & $*$ & 16.5206 & 3916 & 5.6260 & 63 \\
\hline 3 & & 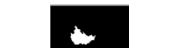 & 12.4948 & 2240 & 3.4885 & 38 \\
\hline
\end{tabular}

tumour segmentation. The following are the main reasons to choose a median filter for brain tumour detection:

- It helps to remove the noise by preserving edge information.

- Basically the median value is calculated by using the values of pixels. Hence it does provide unrealistic pixel value, hence it preserve the edge information safely.

- It uses the non-linear digital filtering method.

The median of a pixel is calculated by using the subsequent steps. Arrange all the pixel value in ascending order, which are neighbours to the considered pixel. Calculate the mean value for the arranged value. When the number of pixels is odd, then the centre values of the pixel are median, for even number of pixel average number of the two centre pixel values is calculated to find the median value of the particular pixel.By using median filtering the noise is removed from the gray scale MRI image as shown in Figure 2. Hence the quality of the image is improved by preserving edge information.

Skull Removal
The second step for preprocessing is skull removal. This is the elementary and essential process to take away the non-brain tissues like fat, scalp, skin, muscle, bones, hair, etc. The proposed research applies morphological segmentation. By using erosion and dilation process the skull removal is performed on gray level MRI image. The following Figure 3 shows the image after elimination of unwanted portion.

\section{Implementation Kisfcm Algorithm}

The process is started by assigning the number of cluster $\mathrm{K}$ and maximum iteration.

Step 1: For this algorithm the $\mathrm{K}$ value is considered as 2 , iteration is considered as minimum value as 100 .

Step 2: Find the cluster centre using the following formula:

$$
m \mu=\frac{(1: k) * m}{k+1}
$$

$K$ (number of clusters $)=2 ; m \mu=$ initial means $m=$ maximum number of iterations

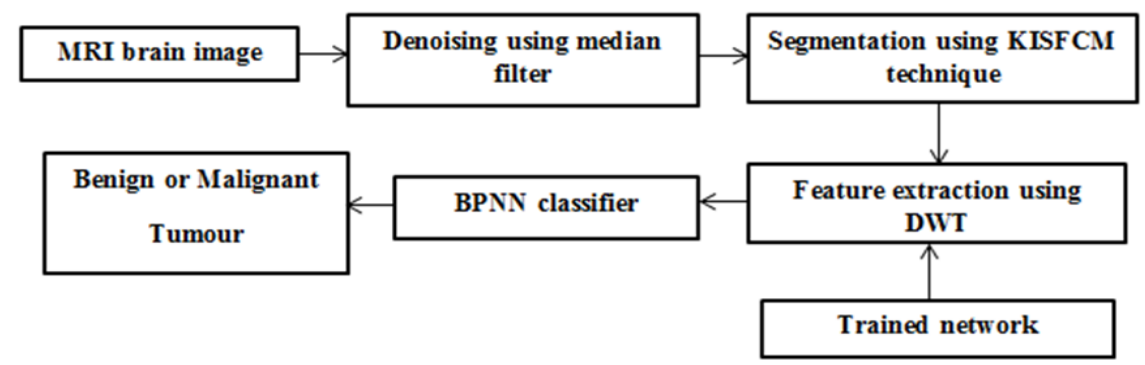

Figure 1. Framework of the Proposed KISFCM Algorithm Along with BPNN Classifier 


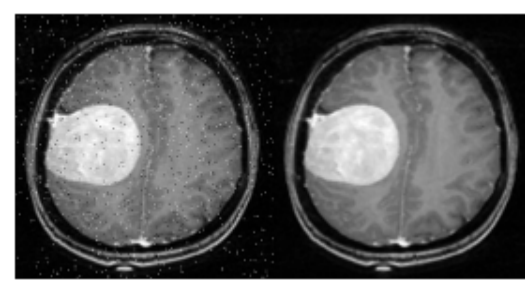

Figure 2. Removal of Noise Using a Median Filter

Step 3: The process begins with by comparing all the pixels with the cluster centre to find the minimum Euclidean distance, then it is pushed to a particular cluster.

Step 4: This process is repeated until the centre convergence.

Step 5: After $\mathrm{k}$ means clustering some of the points dispersed and far away from the cluster centre. As a result of this image again undergone to perform the accurate segmentation, the partially segmented images are undergone for clustering process. Hence the new cluster centres, cluster points and scattered points are labelled into the new loop with the new cluster value 4 . Further the new cluster centre and membership values are updated. Next the data points are re-clustered with new centroids and assign the membership value of the data points, which has the shortest distance between the centroid and data points again. Estimate the objective function. This process is repeated until the cluster centre converges. Morphological operation can be performed on the segmented image in order to extract the tumour portion from the background. The segmented image has to obtain by using erosion operation. In which disk type structuring element is used to get the tumour portion alone. Region props (BW, properties) which helps to calculate the tumour area in the most effective manner within minimal time. After performing the hybrid segmentation the segmented result is shown in Figure 4.

The following Table 1 shows the SFCM output for images with different parameters like area, defected cells, time and iterations.

Next Table 2 shows the different stages of results for KISFCM (K Means Integrated with Spatial Fuzzy C means algorithm).

Table 1 and Table 2 compares the time required for SFCM and KISFCM techniques. In KISFCM method time is reduced and number of iterations required to complete the segmentation process is also reduced. The computation time requirement for SFCM,KISFCM algorithms are shown in Figure 5

\section{Feature Extraction}

To train the BPN network, it is necessary to remove

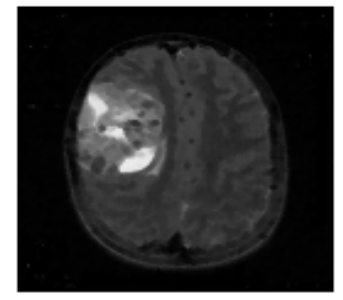

Figure 3. Image after Skull Removal Using K Means Segmentation

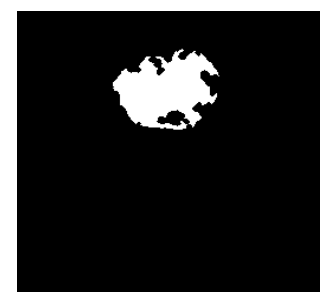

Figure 4. Segmented Image of KISFCM

the different features like Mean, standard deviation, Entropy, Contrast, Correlation and energy from the tumour segmented image. The wavelet transform is one of the important tools to extract the features. Among many types of Haar wavelet transform is one of the simplest type. The proposed work uses Discrete wavelet transfom based harr wavelet is used. The DWT decompose the image into four subbands like LL, LH, HL, HH. The noise present in the image is removed by using thresholding and IDWT helps to get the denoised image.

\section{Classifier Implementation}

Back propagation network follows the systematic procedure to train the multilayer artificial neural network. BPN changes the weights in the feed forward manner, using differentiable activationunctions in order to [rovide an efficient classification. The BPN has three layer like the input layer, hidden layer and the hidden layer. The total number of nodes in the input layer is 7 , it denotes the various features extracted from Region of interest. The number of nodes in the hidden layer is 4 , number of nodes in the output layer is 1 . The process is started by applying the input data set to the input layer, it creates the weighted sum of the output at $\mathrm{j}^{\text {th }}$ node in the hidden layer

$$
\mathrm{Net}_{\mathrm{j}}=\sum w_{i j} x_{i}+\theta_{j}
$$

$\theta_{\text {. }}$ refers the weighted value of the bias node.

The value is always being equal to one. The network is trained by giving the bias value for the node. The neuron firing is happened by giving the sigmoid type of activation function to help the input layer. The activation function generates the result at the output node $\mathrm{k}_{\text {is }} \mathrm{O}_{\mathrm{k}}$ and the target output is denoted as $t_{k}$. Estimate the difference between the actual and target output using the following formula

$$
\Delta_{k}=\mathrm{t}_{\mathrm{k}}-\mathrm{O}_{\mathrm{k}}
$$

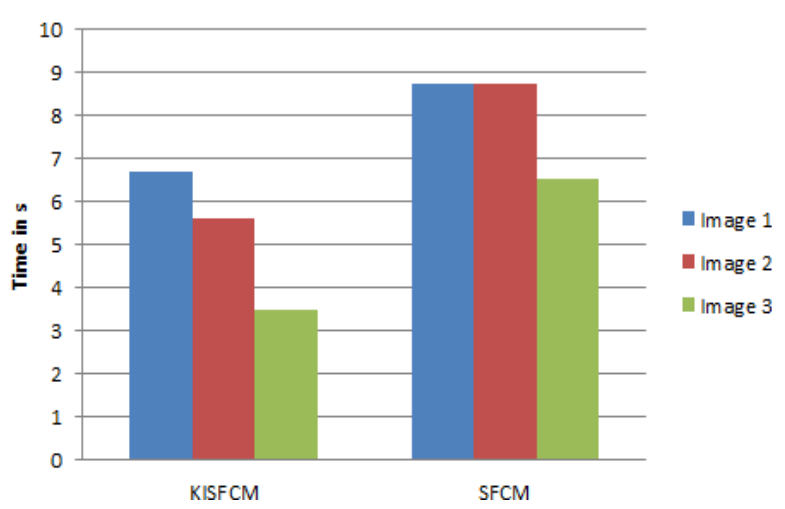

Figure 5. Performance Analysis on KISFCM, SFCM 
Table 3. Extracted Features of Normal MRI Brain Images Using DWT

\begin{tabular}{lcccccc}
\hline Images/ Features & Mean & Standard deviation & Entropy & Contrast & Correlation & Energy \\
\hline 1 & 1.2417 & 16.4258 & 0.0539 & 0.0360 & 0.9399 & 0.9870 \\
2 & 0.9776 & 14.3836 & 0.0426 & 0.1246 & 0.7257 & 0.9870 \\
3 & 0.6113 & 11.9167 & 0.0273 & 0.0585 & 0.7827 & 0.9933 \\
4 & 0.4913 & 10.8738 & 0.0212 & 0.0525 & 0.7383 & 0.9948 \\
5 & 0.3927 & 9.4463 & 0.0185 & 0.0706 & 0.5870 & 0.9951 \\
\hline
\end{tabular}

Table 4. Extracted Features of Abnormal MRI Brain Images Using DWT

\begin{tabular}{lcccccc}
\hline Images/ Features & Mean & Standard deviation & Entropy & Contrast & Correlation & Energy \\
\hline 1 & 14.8844 & 55.0689 & 0.3600 & 0.1036 & 0.9835 & 0.8699 \\
2 & 4.3586 & 31.7704 & 0.1329 & 0.0736 & 0.9588 & 0.9621 \\
3 & 101.5625 & 86.4825 & 0.9563 & 0.3468 & 0.9849 & 0.5542 \\
4 & 4.8523 & 34.5557 & 0.1378 & 0.1561 & 0.9164 & 0.9587 \\
5 & 92.7775 & 79.5200 & 0.9112 & 0.3918 & 0.9817 & 0.5542 \\
\hline
\end{tabular}

$t_{k}$ - target output for the node $k$ in output layer

The Back propagation neural network (BPNN) has three layers. The weight matrices W1, W2 is used to connect the input layer to be hidden layer and hidden layer to the output layer. The network is trained using log-sigmoid activation function having the learning rate of 0.998 to evaluate the feature to classify the MRI brain images. El-Dahshan et al., (2014) illustrated the BPN is to reduce the error at output nodes. Following equation helps to estimate the error $\mathrm{E}$ at all points

$$
\mathrm{E}=\frac{1}{2} \sum \sum\left(t_{k}-O_{k}\right)^{2}
$$

Islam et al., (2013) discussed the extracted features from the Denoised MRI brain image is relates the texture properties of the brain image. The extracted features are acting as an input to train the feed forward BPN network. The accuracy of the proposed system is improved when compared with the existing system. Because the existing method lost the edge information due to shift invariant property. Arizmendi et al., (2012) proposed method is capable to perform fast texture characterization and it is a very flexible method.It is found that the directional features extracted from LH and HL sub bands of the wavelet transform, which gives information along the horizontal and vertical directions respectively, are more efficient at characterizing changes in the biological tissue.

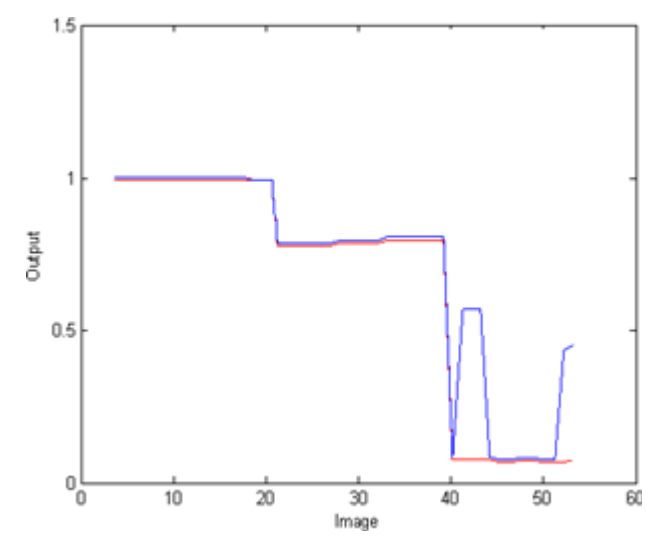

Figure 6. Graph on Target Vs Estimated Output
The five factors Mean, standard deviation, Entropy, Contrast, Correlation and energy have been taken into account for classification of normal and abnormal brain images. The following Table 3 gives details of the various features extracted from normal brain images.

The following Table 4 gives the various features extracted from abnormal brain images.

The following graph shows the performance analysis of brain images. From Figure 6 it is evident in the abnormal brain images has a close association between target and predicted values (range 0.9 to 1.5) and for normal brain images the match between target and predicted values is in the range of 0.3 to 0.89 and is represented in the Figure 6 . Classification efficiency is $93.28 \%$. The efficiency can be improved using an extensive database.

\section{Performance Analysis}

There is a common procedure to evaluate the error rate by using the true and false positive and true and false negative.

$$
\begin{aligned}
& \text { Sensitivity }=\frac{\mathrm{TP}}{(\mathrm{TP}+\mathrm{FN})} \\
& \text { Specificity }=\frac{\mathrm{TP}}{\mathrm{TN}+\mathrm{FP}}
\end{aligned}
$$

Normally the accuracy of the classification can be described as follows

Accuracy $=\frac{\text { Number of correctly classiifed recorded }}{\text { Total records in the test }}$

True positive (TP): The test result is positive when any abnormality present in clinical studies

True negative: Test result is negative when no abnormality is identified

False positive: The test result is positive in the absence of abnormality

False negative: The test result is undesirable in the presence of abnormality. The performance of the algorithm for various images is analysed with Root Mean square, Peak Signal to Noise ratio value.

Validation 
The segmented images using KISFCM were compared to the ground truth cases. The performance analysis was performed to compare the data with ground truth image. The performance analysis PSNR,MSE (Mean Square Error) is calculated from segmentation. The research work uses BRATS data base has multi contrast MRI scans of glioma patients. For every patient T1,T2, FLAIR images are available. All the data are opened by MIPAV and converted to .JPG extension.

In conclusion, the research work is performed using three different types of algorithm. Among the entire algorithm the proposed hybrid segmentation based algorithm provides the best result and provide the accurate calculation of area and time when compared with other two algorithms. Because it integrates the two algorithms, namely $\mathrm{K}$ - means segmentation and spatial fuzzy C-means algorithm, which integrates the distinct features of the two methods.

From the review of various articles it can be identified that the integration of two algorithms leads good result. From this motivation the research work combines the two different algorithms, namely SFCM and K means algorithm and it is called as KISFCM. K means algorithms detect the tumour as faster than FCM, but it provides good result for only the smaller value of $\mathrm{K}$. The next algorithm FCM is used to find the tumour cells that are not connected by K-means. FCM is also not considering the spatial characteristics of brain images. Because this kind of characteristics is very important to classify the complex structures. But it takes long time to perform segmentation. Hence the research work integrates the $\mathrm{K}$ means with SFCM is called KISFCM to get accurate area detection of various algorithms are represented on the following plot in terms of area and time. features. The extracted features are used by the BPNN to do the classification of tumours. The classification efficiency of the network is $87 \%$. This can be improved in this research work with the help of DWT extraction. The extracted features are used by BPNN to provide the improved classification efficiency of $93.28 \%$.

\section{References}

Abdel-Maksouda E, Elmogyb M, Al-Awadi R (2015). Brain tumor segmentation based on a hybrid clustering technique. Egypt Inform J, 16, 71-81.

Arizmendi C, VellidoA, Romero E (2012). Classification of human brain tumours from MRS data using discrete wavelet transform and bayesian neural networks. Exp Sys Appl, 39, 5223-32.

Ciulla C, Veljanovski D, Shikoskaa UR, Risteski FA (2015). Intensity curvature measurement approaches for the diagnosis of magnetic resonance imaging brain tumors. $J$ Adv Res, 6, 1045-69.

Clatz O, Sermesant M, Bondiau PY, et al (2005). Realistic simulation of the 3-D growth of brain tumors in MR images coupling diffusion with biomechanical deformation. IEEE Trans Med Imaging, 24, 1334-46.

Dimitri VDV, Unser M (2008). False discovery rate for wavelet based statistical parametric mapping. IEEE J Sel Top Signal Process, 2, 897-905.

El Dahshan ESA, Mohsen HM, Revett K, Salem ABM (2014).
Computer-aided diagnosis of human brain tumor through MRI: A surveyand a new algorithm. Exp Sys Appl, 41, 5526-45

Hamamci A, Kucuk N, Karaman K, Engin K, Unal G (2012). Tumor-cut segmentation of brain tumors on contrast enhanced MR images for radiosurgery applications. IEEE Trans Med Imaging, 31, 790-804.

Islam A, Syed MSR, Khan MI (2013). Multifractal texture estimation for detection and segmentation of brain tumors. IEEE Trans Biomed Eng, 60, 3204-15.

Ism A, Direkoglu C, Sah M (2016). In proceedings of 12th international conference on application of fuzzy systems and soft computing, August 29-30, Review of MRI based brain tumor image segmentation using deep learning methods, Vienna, Austria.

Juang LH, Wu MN (2010). MRI brain lesion image detection based on color-converted K-means. Scince Direct Measurement, 43, 941- 9.

Logeswari T, Karnan M (2010) Improved implementation of brain tumor detection using segmentation based on soft computing. J Cancer Res Exp Oncol, 2, 6-14.

Madhukumar S, Santhiyakumari N (2015). Evaluation of $\mathrm{k}-$ Means and fuzzy C-means segmentation on MR images of brain. Egypt Soc Radiol Nuclear Med, 46, 475-47.

Matthew CC, Lawrence OH, Dmitry BG, et al (1998). Automatic tumor segmentation using knowledge-based techniques. IEEE Trans Med Imaging, 17, 187-201.

Mohana J, Krishnavenib V, Yanhui G (2014). A survey on the magnetic resonance image denoising methods. Biomed Signal Process Control, 9, 56-69.

Ramathilagama S, Pandiyarajan R, Sathy A, Devi R, Kannan SR (2011). Modified fuzzy c-means algorithm for segmentation of T1- T2 weighted brain MRI. J Comput Appl Math, 235, 1578-86.

Sachdeva J, Kumarb V, Gupta I, et al (2016). A package SFERCB Segmentation, feature extraction, reduction and classification analysis by both SVM and ANN for brain tumors. Appl Soft Comput, 47, 151-67.

Xiang Z, Dazhi Z, Jinwen T, Jian L (2002). A hybrid method for 3D segmentation of MRI brain images. Signal Process, 1, 608-11.

Xing F, Xie Y, Yang L (2016). Automatic learning-based framework for robust nucleus segmentation. IEEE Trans Med Imaging, 35, 550-6.

Yang G, Nawaz T, Barrick TR, Howe FA, Slabaugh G (2015). Discrete wavelet transform based whole spectral and sub spectral analysis for improved brain tumor clustering using single Voxel MR spectroscopy. IEEE J Biomed Health Inform, 62, 2860-6.

Zhang Y, Dong Z, Phillips P, et al (2015). Exponential wavelet iterative shrinkage thresholding algorithm for compressed sensing magnetic resonance imaging. Inform Sci, 322, 115-32.

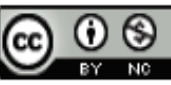

This work is licensed under a Creative Commons AttributionNon Commercial 4.0 International License. 